thus unite with observed facts in indicating that this method is one of extreme value in local malignant growths.

It would be interesting to ascertain the amount of mercury, by weight, that can be carried into a growth per milli-coulomb, also the distance which it will traverse per volt-hour. I find a table in Lodge's work which gives the cataphoric speed of seven substances, of which hydrogen is the most rapid, traversing an electrolyte at the rate of 1.08 centimeters per hour per volt. Lithium is the slowest, at 0.094 centimeter per volt-hour. Taking 0.1 centimeter per volt-hour as a probable figure for mercury, it would mean that 100 volts of current would cause mercury to travel 10 centimeters per hour, or only 1.4 centimeters in ten minutes. These facts have much practical importance, indicating that we must have a sufficient duration of the current to secure proper penetration, and that amperage alone, while governing the amount and density of the penetration, is incapable of increasing its extent without adding a time element. Since seeing this table of Dr. Lodge I have been convinced that some of my applications have had an insufficient duration. The exact cataphoric speed of mercury should be determined.

The practical details of this method, as applied by me to the cure of cancer, consists of two selective procedures, the gradual and the rapid, adapted to different cases and conditions of malignancy. The gradual method is simply an intensification of a method used by me daily in the cure of obstinate catarrh of the uterus and similar conditions, in which an amalgamated zinc or gold eleotrode is applied to the ulcerated surface and as much mercury driven in, by daily applications, as the moderate pain produced will permit. This is adapted to incipient cancers and very bad inoperable cancers in which the rapid method is injudicious.

In the rapid method the patient is thoroughly anesthetized; two or three large indifferent pads are placed under the back and connected with the negative pole of the battery. To the positive pole a multipleconnection cord is attached which is to be connected to one or more lancet electrodes, when the latter have been inserted into the growth. Usually an opening must be made into the tumor for the insertion of the electrodes. After being troubled in several cases with the bleeding which this causes, the only bloodloss of the whole method, I attached a separate steel spear electrode to the negative pole and, with this made an electrolytic opening of the proper size. This requires reversing the current for the first insertion, but after the first electrode is in place the current may be turned off at the controller, reversed, and turned on in the proper direction, with one electrode acting. The spear may now be attached afresh to the same binding post that is conneeted to the pads, and used to make additional openings, into which fresh zinc electrodes are placed and put in connection with the multiple-connection positive cord. No blood will be lost or obscure the field of action.

Five hundred milliamperes is the greatest current so far used in this monopolar method, as this amount has depressed the breathing by a tetanic contraction of the diaphragm. This was doubtless due to the fact that one of my dispersing pads was on the abdomen and the other on the back. In the next case I shall place both pads on the back.

In two cases I have hurried the local dissemination. of the mercury, by placing a cotton-covered disc, saturated with liquor potassii arsenitis on the center of the tumor, as the negative pole, cutting out the dispersing pads. The application now being bipolar and mainly local. the current was increased to 1,000 milliamperes. It will be noted that this application produced a valuable cataphoric action at each pole, mercury going in at the anode and arsenic at the cathode.

After sufficient local effect has been produced the application is terminated and the patient put to bed. At the end of twenty-four hours the slough that has been produced, will be found to be surrounded by an area in which the cancerous manifestations have been quelled entirely, or partially, in accordance with the thoroughness of the application, without the sustaining tissues being necrosed. The slough, itself, is largely aseptic and will come away in about twelve days, permitting us to determine whether a repetition of the process is necessary. The cavity left fills up readily by granulation.

Note.-At date of publication the two cured cases mentioned continue well. Three additional cases of completely successful removal of cancerous tumors by the method have since been added to the list.

\section{THE NEED OF DENTAL INSTRUCTION IN MEDICAL SCHOOLS.}

Presented to the Section on Stomatology, at the Forty-eighth Annual Meeting of the American Medical Association, beld at Philadelphia, Pa., June 1-4, 1897.

BY EDWARD BRANIGAN, D.D.S. BOSTON, MAss.

There is a great need of dental instruction in medical schools. Physicians should know more about the teeth than they now do, and when medical students are given a better knowledge of the teeth and of the many disturbances they cause, then will medical science do more for humanity than it is now doing. More than a dozen years spent in looking into the mouths of my neighbors, at the rate of hundreds of mouths a week, has revealed to my observation so many instances of improper treatment of diseases caused by the teeth, that I have long felt it my duty to protest, and.I now take advantage of the opportunity given me to put my protest into the relation of experiences that have come to me so many times that I put them forth as cold facts. I shall confine myself to the narration of a few of the more common instances of mismanaged treatment of diseases caused by the teeth. The evils I speak of are common.

Abscesses caused by pulpless teeth are still poulticed and opened on the face by medical men. I have seen many hundreds of these, for this treatment never effects a cure, and in the course of time it dawns upon the patient, if not upon the doctor, that dental assistance is needed. How much better it would have been for the patient to have been sent to the dentist at first and so avoided days and weeks of misery and the necessity of carrying a hideous scar for life. People are still being stabbed on the face and neck because a third molar sometimes causes trouble in trying to get up into the mouth where it belongs. The medical treatment for these simple abscesses is sometimes varied a little. The sufferer may be kept in bed for a time, with temperature and pulse watched, and medicine changed at intervals, according to the symptoms. This sometimes happens when the old tooth-root, at the bottom of the trouble, is so loose that the owner 
could pull it ont with two fingers. Pus from an abscessed tooth is frequently discharged into the antrum. The extraction of the tooth, followed by proper treatment, would rob the medical profession of many patients who are supposed to suffer from, and are treated for, catarrh. Serious throat diseases are frequently caused by pulpless teeth and sometimes by unerupted or retained teeth. All throat specialists are not aware of this fact.

A more intimate knowledge of the mouth and teeth would help other specialists, aurists, for instance. Severe and painful inflammations of the ear, caused by teeth, are common. The removing of fillings from pulpless teeth has made many sufferers from improper aural treatment happy. Unerupted wisdom and occasionally unerupted supernumerary teeth are the cause of many obscure ear troubles. Next to the dentist, no one needs dental knowledge more than the aurist. Oculists are now tracing a variety of diseases of the eye back to the teeth, but they have not yet found out all the mean things a diseased tooth can do. Many sufferers from irritation of the eye, backed up by an inflamed tooth, do not get to the oculist, and if the general practitioner knew more about the teeth as a cause of disease, he would not in so many of these cases prescribe an eye-wash, or glasses, or a tonic; he would send the patient to a dentist.

A tumor of the nose may be caused by a dead toothpulp. Treatment of the tumor without a knowledge of the cause is not calculated to make the owner of the nose happy. The victims of so-called neuralgia, who after weeks and months and even years of antineuralgic drugging, die or find relief at the hands of someone who knows something about teeth, are countless. Thousands of people are today being drugged for neuralgia who are really suffering from some form of dental irritation. A pulp-stone no larger than a pin-head can bring about so much pain and loss of sleep, appetite and health that nervous prostration. may be, and often is, the end of an affair that a pair of forceps would have ended in a moment.

Bicycle riding has given relief to many sufferers from dyspepsia, but many more dyspeptics would get better if their medical advisers insisted upon their having all their lame and tender teeth cared for. Among the hundred or two of mouths opened every morning for my inspection, I never fail to find a num. ber showing evidence of being used on one side only, and in many of the mouths the front teeth are the only ones that do any work.

Physicians should also take into consideration the constant presence of pus in the mouths of many of their patients. They do not all do this. I find pus in physician's mouths about as often as I find it in the mouths of other classes of patients.

The authorities in charge of the management of hospitals should know more of dentistry. If they did, the nurses and other hospital attendants would know how to cleanse a patient's mouth, and the hospital surgeon would know how to look into a patient's mouth intelligently. The removal of half or a whole jaw for necrosis, would not take place when the extraction of a few roots and the use of an antiseptic mouth-wash would effect a cure. When hospital surgeons know more about the mouth and jaws we shall not be obliged to meet people who have been dismissed from hospitals after some weeks of treatment for broken jaw, with the fracture ununited or with the union in so erratic a form that the sufferer can not close his jaws. When some one about the hospital is able to examine a mouth we shall not be called on to help the man who takes his broken jaw away from the hospital after having been assured that he will be all right in a few days if he puts a poultice on his bruised face and takes a few pills. It is a fact that some surgeons can not locate a fracture of the jaw, and it is also a fact that when located, fractured jaws are sometimes treated by being bound together with a four-tailed bandage. and then, as a happy afterthought, a couple of front teeth are pulled out to make a hole through which to tuck the pationt's nourishment.

After dental instruction is given to medical students, the army and the navy will get a little much needed dental service. They get very little now, although hospital stewards are supposed to be able to pull teeth.

The medical directors of public institutions will find a vast amount of good work ready for them when they realize the importance of having dental service given to those in their charge. A few years ago I discovered that not one of the 500 children in one of the public institutions in my city had a tooth-brush. The children of that institution are now supplied with toothbrushes, but I fear there are many similar institutions in the world where the tooth-brush is never seen and the dentist never heard of. I am sure that this evil will be corrected very soon after dental instruction is given to the future medical directors of public institutions for the care of the poor.

\section{PATHOLOGIC CONDITIONS OF THE PHAR YNX AND CONTIGUOUS STRUCTURES DURING EARLY CHILDHOOD, PRIME FACTORS IN THE ETIOLOGY OF MALFORMED MAXILLAE AND IRREGULAR TEETH, ETC.}

presented to the Section on Stomatology, at the Forty-eighth Annual Meeting of the American Medical Association, held at Philadelphia, Pa., June 1-4. 1897.

BY WILLIAM A. MILLS, D.D.S. BALTIMORE, MD

The physiologic functions of the nasal cavities are as follows: "They elevate the temperature of the inhaled air, give it moisture, and purify it by arresting all particles of dust and other substances which it may contain;" they act as resonance cavities for the voice and are also the seat of the sense of smell.

A professional experience of more than twenty-five years, has taught me that any inflammatory lesionsround in children between the ages of 4 and 12 yearswhich obstruct these natural conditions, are in the majority of cases, the chief agents in the causation of malformed jaws, and the consequent abnormal alignment of the teeth. (To the same cause can be attributed beaked or hooked noses, so often found in connection with these deformities.) I have reached this conclusion after many years of ceaseless endeavor to trace out cause and effect. Others may theorize differently, but theory alone will not stand, unless proven clinically correct.

I cite the following case in my practice:

A male child over 7 years old was brought to have a tooth filled, which was supposed to cause pain in the right ear and the right angle of the inferior maxilla.

The patient presented the following clinical conditions: Almost complete obstruction of the nasal cavities; face pale, ane. mic and haggard ; features contracted at the angles of the jaws and beneath the malar processes, giving the characteristics of the mouth-breather or "dog-face," as Dr. Cathell terms them. I 